the earliest prokaryotes as introns-early requires, or did it recently move to this site? In a vigorous advocacy of intronslate, J. Palmer (Indiana Univ.) discussed the unpublished work of V. Walker and M. Tyshenko (Queen's Univ., Ontario) which, he argued, suggests a recent ancestry. Other than Culex and its close relative Aedes, Walker and Tyshenko have found no species with an intron in the 'Gilbert position', not even in other mosquitoes outside the Aedes/Culex subfamily. Surely, Palmer argues, a phylogenetic tree in which all but one group does not have a character is most parsimoniously interpreted as evidence of gain in that one, rather than losses in all other taxa. With an absence of spliceosomal introns in all prokaryotes ${ }^{6}$ the same logic can be more generally applied. The finding of rare intron positions simply adds to the number of losses (or slides) that must be invoked.

Although this argument may make gain more parsimonious than loss, if introns are only ever lost, every intron position in every gene must at some point be found in one species or group only. So intronsearly is not incompatible with instances of unique intron positions. The above mode of analysis cannot then disprove intronsearly, it just renders it unparsimonious.

Work by Palmer himself and his colleague J. Logsdon also counts against introns-early. The two have established ${ }^{6}$ that across eukaryotes there is a great deal of variation in the average number of spliceosomal introns per unit kilobase of coding DNA (from none to six introns per kilobase). They have now gone on to examine the titre of spliceosomal introns in genes that had been acquired from organelles but that currently reside in the nucleus. They find a good correspondence between the average intron number per kilobase of coding DNA for nuclearlocated, organelle-derived, genes and the average for genes in the same organism that are ancestrally of nuclear origin.

This provides strong evidence that intron density, at least of the spliceosomal introns of nuclear genes, is dependent upon some feature of the organism that contains them. It is compatible with the introns-late model, as it assumes that introns can come and go at an appreciable rate, but problematic for introns-early. Spliceosomal introns have yet to be found in the putative sister groups of both mitochondria (alpha-purple bacteria) and chloroplasts (cyanobacteria). So to maintain that the positions of introns in the mitochondrial- and chloroplast-derived genes are ancestral, one has to argue that cyanobacteria and alpha-purple bacteria both had high titres of introns but subsequently lost them. This again is not impossible - just unparsimonious.

The new comparative data also provide the basis for tackling a different question: what aspects of organismic biology can explain the variation in intron density? At least three sets of models attempt to account for this variation. First, selection in favour of an increased replication rate could select for reduced intron density. Second, introns may regulate the expression of genes involved in complex developmental strategies and their titre should hence be dependent upon developmental complexity ${ }^{7}$.

Third, sexual reproduction could provide the conditions for selection favouring increased intron density, for one of two reasons. If introns are selfish DNA they may spread in a population even if deleterious, but only if outbreeding events are regular enough ${ }^{8}$. Alternatively, introns may be a defence against the deleterious consequences of recombination. What if, as may indeed be the case, crossing-over causes flanking mutations (ref. 9 but see also 10)? Recombination within a gene could be deleterious but recombination in an intron would not. Alternatively, recombination may tend to cause chromosomal rearrangements if it occurs at illegitimate sites. Introns could tend to break up homologous sequences that occur at chromosomally non-homologous sites so preventing mispairing ${ }^{11}$

The three classes of models interpret the data differently. All can cite as support both the absence of spliceosomal introns in prokaryotes and organelles, as well as the low densities in unicellular eukaryotes. The replication rate model need simply invoke the idea that the above are all $r$-selected, the sex/recombination models need point to the fact that they are mostly clonal or with only occasional sex and the developmental regulation model need point to the developmental simplicity co-ordinated by the genomes concerned.

Through inter- and intra-genomic comparative analyses, we may be able to tease the forces apart. We may then find out what, if anything, introns and sex have in common, and just how unreasonable it is, given the absence of spliceosomal introns in prokaryotes, to suppose that ancestral bacteria were ever intron rich.

Laurence $D$. Hurst is in the Department of Genetics, University of Cambridge, Downing Street, Cambridge CB2 3EH, UK.

\footnotetext{
1. Alberts, B. et al. Molecular Biology of the Cell, 3rd edn (Garland, New York, 1994).

2. Gilbert, W. \& Glynias, M. Gene 135, 137-144 (1993)

3. Stoltzfus, A. et al. Science 265, 202-207 (1994)

4. Gilbert, W., Marchionni, M. \& McKnight, G. Cel/ 46 151-154 (1986)

5. Tittiger, C. Whyard S. \& Walker, V.K. Nature 361 470-472 (1993)

6. Palmer, J. D. \& Logsdon, J. M. Jr Curr. Opin. Genet. Dev. 1, $470-477$ (1991)

. Mattick, J. Curr. Opin. Genet. Dev. (in the press)

8. Hickey, D. A. \& Benkel, B. J. theor. Biol. 121, 283-291 (1986).

9. Magni, G. E. J. cell. comp. Physiol. 64, 165-172 (1964)

10. Lawrence, C. W. Adv. Genet. 21, 173-254 (1982)

11. Kricker. M. C., Drake, J. W. \& Radman. M. Proc. natn. Acad. Sci.U.S.A. 89, 1075-1079(1992).
}

\section{Blaze of colour}

LAST week Daedalus suggested that a monolayer of oxygen adsorbed on a combustible surface could be explosive. If the top molecular layer of the surface could be made to burn in the adsorbed oxygen, the resulting two-dimensional explosion would propagate over the surface by Rayleigh surface waves. It could be used for cleaning and for controlled surface erosion.

He is now scaling up the idea. Conventional explosives need enough energy-density to propagate a blast in three dimensions. An explosive too feeble to explode in bulk might still manage to do so along a surface. One candidate is under-nitrated cellulose, used in many lacquers and varnishes. With the aid of special shaped-charge detonators designed to explode sideways, Daedalus is trying to explode the nitrocellulose varnish on old sideboards, television sets, filing cabinets, cars and so on.

Painters and decorators will await the results with keen interest. The most tedious part of their job is removing the old paint. A nitrocellulose paint could be blown off the wall by direct sideways detonation. Other paints might need to be nitrated first, by brushing on a special chemical paste. Better than any scraper and blowtorch, the explosion would propagate into all the nooks and crannies of the surface, leaving it cleaned and flamed ready for the new paint. This small, distributed explosion should do little structural damage. Its energy would be spread over the whole surface, and its shock would run parallel to that surface, rather than down onto it.

Exploding paint will be quite safe on the wall. Even in a fire it will burn quietly; only the special sideways detonator will make it explode. Every householder will adopt it, looking ahead to the time when it has to be replaced. Ship owners will welcome it - at present many ships are scrapped to avoid the cost of repainting. It will replace the waxes and transport coatings that protect cars during shipment. Once the car reaches the showroom, the retailer will blast the coating off again. The sudden surface heating may even briefly melt the conventional paint underneath, enhancing its gloss. Unscrupulous car owners, however, may use exploding paint as a permanent finish. They will spray their vehicles with an added explodable top layer in some contrasting colour. After an accident or brush with the law, they will blast it off again - it could even be arranged to detonate automatically on impact. The car will change colour instantly, confusing the witnesses.

David Jones 